\title{
Piso de bambu chinês vs. piso de eucalipto brasileiro: estudo de caso comparativo das emissões de gases de efeito estufa no transporte
}

\author{
Chinese Bamboo flooring vs. Brazilian eucalyptus wooden \\ floor: case study of the greenhouse gas emissions by \\ transportation
}

\section{Thiago Zaldini Hernandes}

\begin{tabular}{|c|c|}
\hline & Resumo \\
\hline & $\begin{array}{l}\text { om a disseminação de produtos para construção supostamente mais } \\
\text { sustentáveis, tais como o piso de bambu importado da China, surge a } \\
\text { necessidade de compreender, de fato, o quão mais sustentáveis são tais } \\
\text { opções diante de soluções locais. Analisando apenas as emissões de } \\
\text { gases de efeito estufa relacionados ao transporte, esta pesquisa comparou duas } \\
\text { soluções de acabamento de piso maciço: o piso de bambu chinês e o assoalho de } \\
\text { madeira de eucalipto brasileiro. A análise do saldo de emissão de gases de efeito } \\
\text { estufa demonstrou que o transporte do piso de bambu ao redor do mundo não } \\
\text { consegue sozinho comprometer todo o potencial de sequestro de carbono desse } \\
\text { material. No entanto, sua contribuição para a diminuição do saldo é significativa. } \\
\text { A contribuição do transporte diminuiu em } 28,1 \% \text { o potencial de sequestro do } \\
\text { material bambu contra a diminuição de } 1,4 \% \text { para o material assoalho de madeira } \\
\text { de eucalipto produzido localmente. Ainda, a pesquisa demonstrou que, apesar da } \\
\text { maior massa de material utilizada por metro quadrado de piso acabado, a emissão } \\
\text { de gases de efeito estufa no transporte do eucalipto é cerca de } 8 \text { vezes menor do } \\
\text { que a do piso de bambu. }\end{array}$ \\
\hline & $\begin{array}{l}\text { Palavras-chaves: Bambu. Eucalipto. Piso. Sequestro de carbono. Gases de efeito } \\
\text { estufa. }\end{array}$ \\
\hline & Abstract \\
\hline & $\begin{array}{l}\text { The dissemination of allegedly more sustainable construction products, such as } \\
\text { bamboo flooring imported from China, makes it crucial to understand how much } \\
\text { more sustainable those products actually are when compared with their local } \\
\text { equivalents. By analysing exclusively the emissions of greenhouse gases related to } \\
\text { their transportation, this study compared two floor finish products: Chinese } \\
\text { bamboo flooring and Brazilian eucalyptus wood floor. The analysis of the net } \\
\text { emissions of greenhouse gases demonstrated that in the case of the bamboo } \\
\text { flooring, its transportation across the world alone does not undermine the entire } \\
\text { carbon sequestration potential of the material. However, its contribution to } \\
\text { reducing the balance is significant. The transportation reduced the sequestration }\end{array}$ \\
\hline iago Zaldini Hernandes & $\begin{array}{l}\text { reducing the balance is slgnificant. The transportation reduced the sequestration } \\
\text { potential of the bamboo material by } 28.1 \% \text {, as opposed to a reduction of } 1.4 \% \text { for }\end{array}$ \\
\hline Universidade de São Paulo & the locally produced eucalyptus wood. In addition, the study showed that despite \\
\hline São Carlos - SP - Brasil & $\begin{array}{l}\text { the greater mass of material used per square meter of finished floor, the emissions } \\
\text { of greenhouse gases by the transportation of eucalyptus wood is about } 8 \text { times }\end{array}$ \\
\hline n 02/09/13 & lower than that of the bamboo flooring. \\
\hline Aceito em $24 / 12 / 14$ & words: Bamboo. Eucalyptus. Floor. Carbon sequestration. Greenhouse gases. \\
\hline
\end{tabular}

HERNANDES, T. Z. Piso de bambu chinês vs. piso de eucalipto brasileiro: estudo de caso comparativo das emissões de 7 gases de efeito estufa no transporte. Ambiente Construído, Porto Alegre,v. 15, n. 1, p. 7-16, jan./mar. 2015. ISSN 1678-8621 Associação Nacional de Tecnologia do Ambiente Construído. http://dx.doi.org/10.1590/S1678-86212015000100002 


\section{Introdução}

A intensificação na busca por soluções mais sustentáveis na indústria da construção civil tem incentivado a disseminação de novas tecnologias ditas "verdes", ou "sustentáveis".

Seguindo essa tendência, o uso de produtos renováveis e/ou com baixa emissão de carbono tem incentivado a disseminação de produtos feitos à base de madeira e similares. Um desses produtos que vêm ganhando destaque é o piso de lâminas de bambu. Esse material, usualmente proveniente de fornecedores chineses, é apresentado por seus revendedores locais como uma alternativa mais sustentável do que as soluções tradicionais de materiais para piso. Esses revendedores defendem tal posição principalmente com base no fato de que o produto:
(a) é renovável;
(b) é durável; e
(c) tem baixa emissão de carbono, por causa de sua origem vegetal.

No entanto, a grande distância de sua região produtora (sudeste asiático) até aqui motivou a análise das emissões de gases de efeito estufa de seu transporte. Afinal, apesar de ter carbono incorporado (e isso ser utilizado como argumento de venda), o quanto o transporte ao redor do mundo compromete seu saldo de emissões?

Para responder a tal pergunta, a análise confrontou o carbono incorporado no material em relação à emissão de seu transporte da China para o Brasil.

Há de se observar que o saldo total do sequestro de carbono de determinado produto deve contemplar as etapas de produção, transporte, uso e seu destino final. Para este trabalho, no entanto, não foi possível ter acesso a detalhes sobre a fabricação do produto, pelo fato de o revendedor do piso de bambu manter sigilo acerca dessas informações. Assim, cabe deixar claro que o escopo deste trabalho trata exclusivamente de calcular o saldo de emissões relativo ao transporte do piso de bambu com vistas a verificar se essa etapa sozinha seria capaz de comprometer o saldo de carbono incorporado no material.

Ainda, com o propósito de permitir uma abordagem crítica realista e comparativa, tal confrontação também foi feita em outro material utilizado para piso, que também é vendido, grosso modo, sob a alegação das mesmas vantagens referentes à sustentabilidade: o piso maciço de eucalipto. Dessa forma, confrontam-se produtos que atendem ao mesmo nicho de mercado.

\section{Materiais e Métodos}

\section{Piso de bambu}

Para este estudo de caso, a empresa fornecedora de piso de bambu escolhida foi a Ecori Pisos e Revestimentos Ecológicos. Para a coleta de dados entrevistou-se o proprietário da empresa, o $\mathrm{Sr}$. Leandro Martins (MARTINS, 2012).

A empresa está localizada em São José do Rio Preto, SP, a $444 \mathrm{~km}$ da capital do estado. Seus produtos são pisos, painéis e móveis feitos com bambu. Todos os seus produtos são provenientes da China. A Figura 1 ilustra a aplicação do piso de bambu.

Os pisos de bambu são apresentados em réguas maciças de $1.840 \mathrm{~mm} \times 126 \mathrm{~mm} \times 12 \mathrm{~mm}$ ou $1.905 \mathrm{~mm}$ x $195 \mathrm{~mm}$ x $14 \mathrm{~mm}$. As réguas de 14 $\mathrm{mm}$ têm um peso de aproximadamente $10 \mathrm{~kg} / \mathrm{m}^{2}$, portanto uma densidade de $714 \mathrm{~kg} / \mathrm{m}^{3}$ ou 0,714 $\mathrm{t} / \mathrm{m}^{3}$. A Tabela 1 apresenta as características dimensionais do piso de bambu selecionado.

Figura 1 - Imagem ilustrativa de aplicação do piso de bambu chinês da empresa Ecori

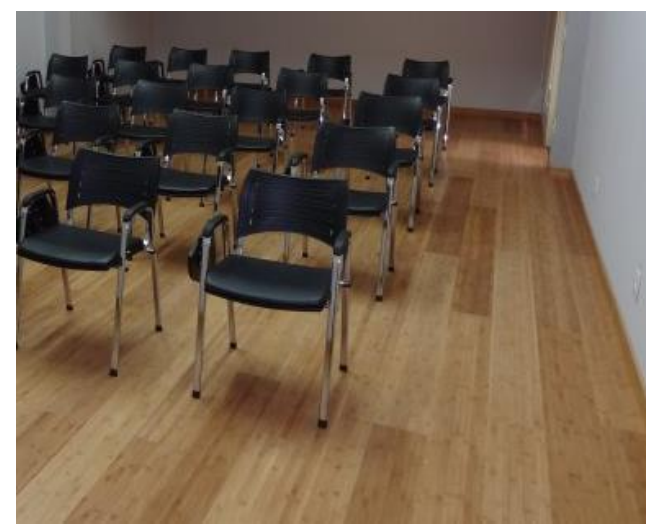

Fonte: Ecori (2015).

8 Hernandes, T. Z. 
Tabela 1 - Características dimensionais dos pisos de bambu de $12 \mathrm{~mm}$ e de $14 \mathrm{~mm}$

\begin{tabular}{|c|c|c|}
\hline & $12 \mathrm{~mm}$ & $14 \mathrm{~mm}$ \\
\hline Comprimento (m) & 1,840 & 1,905 \\
\hline Largura $(\mathbf{m})$ & 0,126 & 0,195 \\
\hline Altura (m) & 0,012 & 0,014 \\
\hline Volume $\left(\mathbf{m}^{\mathbf{3}}\right)$ & 0,00278 & 0,00520 \\
\hline Área $\left(m^{2}\right)$ & 0,23184 & 0,37148 \\
\hline Densidade $\left(\mathrm{kg} / \mathrm{m}^{3}\right)$ & 714 & 714 \\
\hline Peso por peça & 1,986 & 3,713 \\
\hline $\mathrm{kg} / \mathrm{m}^{2}$ & 8,568 & 10,000 \\
\hline
\end{tabular}

Fonte: Ecori (2015).

\section{Piso de eucalipto}

Também foi identificado um fornecedor de piso de eucalipto para este estudo, a Assoalho Ecológico. Para a coleta de dados foi entrevistado um vendedor da empresa e consultada a página da empresa na internet (ASSOALHO..., 2015).

A Assoalho Ecológico está localizada na cidade de São Paulo. Seus produtos são pisos maciços de madeira para assoalhos, tacos e deques. Entre seus produtos está o assoalho maciço de madeira.

Os assoalhos são fornecidos sempre com a espessura padrão de $20 \mathrm{~mm}$ e com larguras de 70 $\mathrm{mm}, 100 \mathrm{~mm}, 150 \mathrm{~mm}$ ou $200 \mathrm{~mm}$. O entrevistado informou que o fornecimento de eucalipto não é proveniente de uma única região, mas é usual que seja comprado no próprio Estado de São Paulo.

Como o eucalipto utilizado é o da espécie citriodora (Eucalyptus citriodora), sua densidade foi considerada como sendo $1.040 \mathrm{~kg} / \mathrm{m}^{3}$ (INSTITUTO..., 2011). A Figura 2 ilustra a aplicação do piso de assoalho de eucalipto.

\section{Sequestro de carbono}

Tanto o bambu quanto o eucalipto sequestram carbono da atmosfera para formar sua massa viva. Para se determinar o potencial de sequestro de carbono do produto em questão, confronta-se a quantidade de carbono armazenada na madeira e no bambu, contra o total de $\mathrm{CO}_{2}$ eq emitido ao longo de sua cadeia produtiva.

O trabalho de Delgado (2011) apresenta uma análise elementar $(\mathrm{CNH})$ tanto do bambu quanto do eucalipto. Nessa análise a variação de carbono entre as duas espécies foi de 2,7\%. Portanto, considerando similar a quantidade de carbono tanto do bambu quanto do eucalipto, utilizou-se a mesma equação de Reis et al. ${ }^{1}$ (1994 apud SILVA,

${ }^{1}$ REIS, M. G. F. et al. Sequestro e Armazenamento de Carbono em Florestas Nativas e Plantadas dos Estados de Minas Gerais e Espírito Santo. In: SEMINÁRIO EMISSÃO X SEQUESTRO DE $\mathrm{CO}_{2}$, Rio de Janeiro, 1994. Anais... Rio de Janeiro: Companhia Vale do Rio Doce, 1994.
2015) para ambos. Segundo essa equação, a quantidade carbono (C) é igual a $50 \%$ do peso da madeira. Assim, tanto $1 \mathrm{t}$ de bambu quanto $1 \mathrm{t}$ de eucalipto tem $0,5 \mathrm{t}$ de carbono.

No entanto, como cada $1 \mathrm{~kg}$ de carbono (C) equivale a $3,67 \mathrm{~kg}$ de $\mathrm{CO}_{2} \mathrm{eq}^{2}$, cada tonelada de bambu ou eucalipto tem armazenados $1.835 \mathrm{~kg}$ de $\mathrm{CO}_{2}$ eq.

\section{Desenvolvimento}

Para a análise do saldo de emissão de $\mathrm{CO}_{2}$ no transporte do piso de bambu em face do transporte do piso de eucalipto, foram estabelecidos parâmetros de comparação com base em suas regiões de origem, destino, tipo de transporte e densidade.

\section{Origens}

O piso de bambu da empresa Ecori é proveniente de um fornecedor do sudeste chinês, mais particularmente da região de Shenzhen. Segundo o proprietário da empresa, o piso é produzido em indústrias próximas das florestas de bambu do sul da China, na região de Shenzhen (Figura 3). A localização exata foi mantida em sigilo pelo entrevistado.

A madeira de eucalipto utilizada pela empresa Assoalho Ecológico não tem um único fornecedor identificado, mas para o escopo deste trabalho assume-se que ela seja produzida em um raio de $500 \mathrm{~km}$ de distância em relação à cidade de São Paulo. Tal distância abrange regiões produtoras de eucalipto: o centro do estado de São Paulo, o sul de Minas Gerais e o norte do Paraná (Figura 4).

\footnotetext{
${ }^{2} \mathrm{O}$ fator de conversão de carbono em dióxido de carbono igual a 3,67 foi obtido pela razão entre a massa molecular do dióxido de carbono $\left(\mathrm{CO}_{2}\right)$ igual a $44 \mathrm{~g} / \mathrm{mol}$ e a massa atômica do carbono $(\mathrm{C})$ igual a $12 \mathrm{~g} / \mathrm{mol}$
} 
Figura 2 - Exemplo de assoalho de eucalipto da empresa Assoalho Ecológico

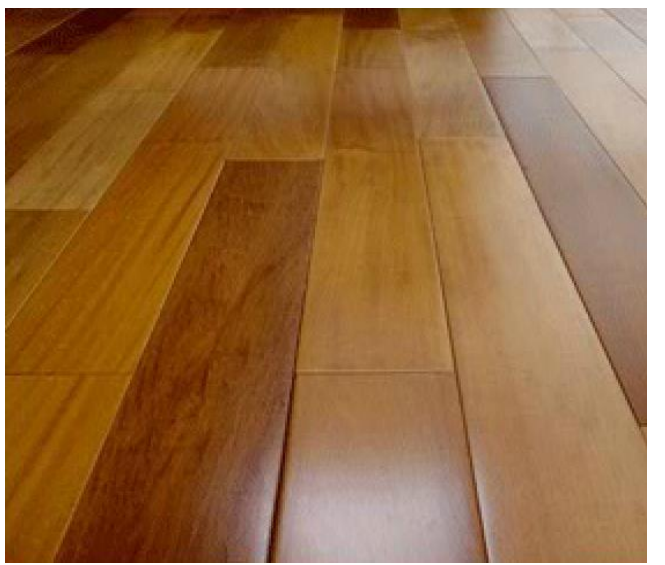

Fonte: Assoalho Ecológico (2015).

Figura 3 - Mapa da República Popular da China que indica a localização da cidade de Shenzhen

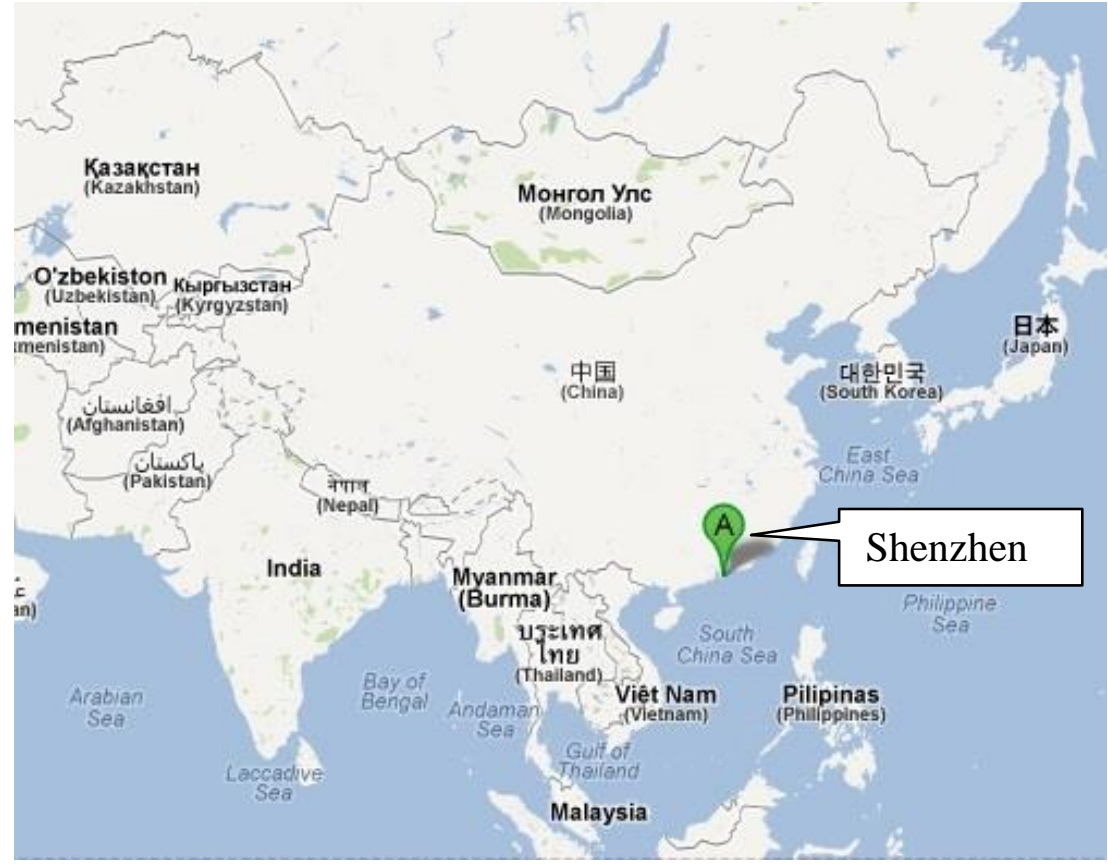

Fonte: Google Maps (2012).

\section{Meios de transporte}

Enquanto o transporte da madeira de eucalipto é realizado por caminhão até seu destino, na cidade de São Paulo, o piso de bambu é transportado por via marítima, em contêineres.

Baseando-se nas rotas marítimas mais prováveis, foi calculada a distância percorrida por um navio cargueiro deslocando-se diretamente de Shenzhen, na China, para Santos, no Brasil. Utilizando a calculadora de rotas marítimas do site SeaRates.com (SEA-RATES, 2015) foi possível estimar a distância de 10.258 milhas náuticas. Essa distância equivale a $18.997,80 \mathrm{~km}$. Para os cálculos realizados neste trabalho, o valor foi aproximado para $19.000 \mathrm{~km}$ (Figura 5).

A trajetória do piso de bambu inclui $19.000 \mathrm{~km}$ (cerca de meia volta ao mundo) de transporte marítimo por cargueiros em contêineres (Shenzhen-Santos), além de mais $500 \mathrm{~km}$ de transporte terrestre em caminhão a diesel (SantosSão José do Rio Preto).

Por sua vez, a madeira do piso de eucalipto tem um transporte presumido de $500 \mathrm{~km}$ do local de plantio até São Paulo, onde é processada, e de lá mais $500 \mathrm{~km}$ até a cidade de São José do Rio Preto, o que totaliza $1.000 \mathrm{~km}$ de transporte, realizado por caminhão a diesel. 
Figura 4 - Mapa das regiões produtoras de eucalipto com indicação de raio de 500 km ao redor da cidade de São Paulo

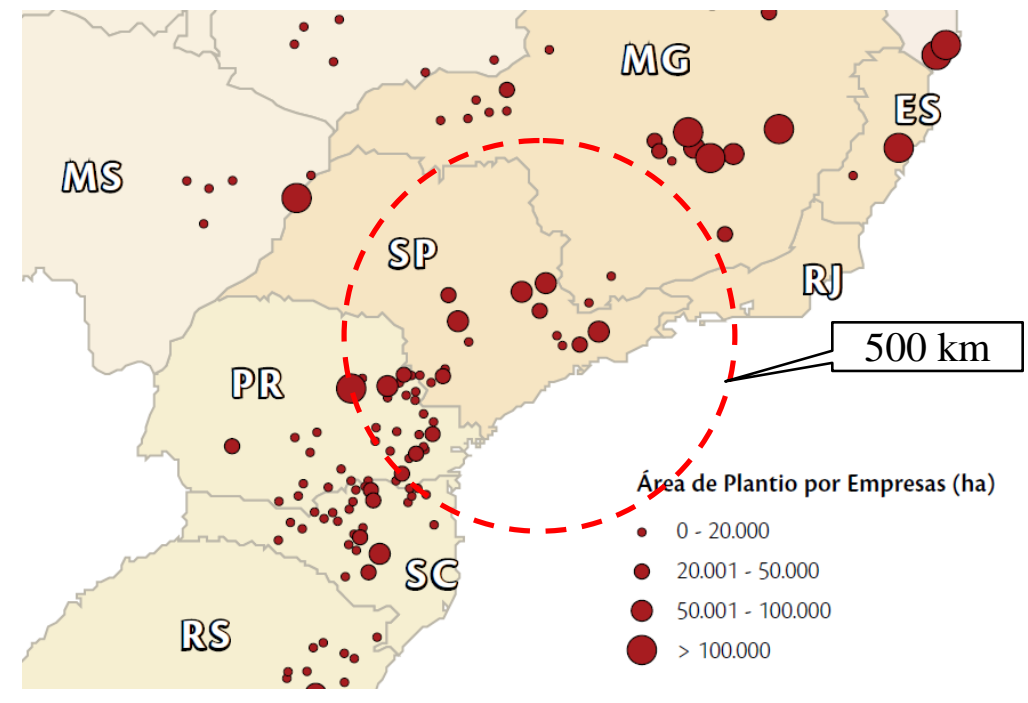

Fonte: Associação Brasileira de Produtores de Florestas Plantadas (2011).

Figura 5 - Rota marítima comercial que indica a distância entre os portos de Shenzhen e Santos

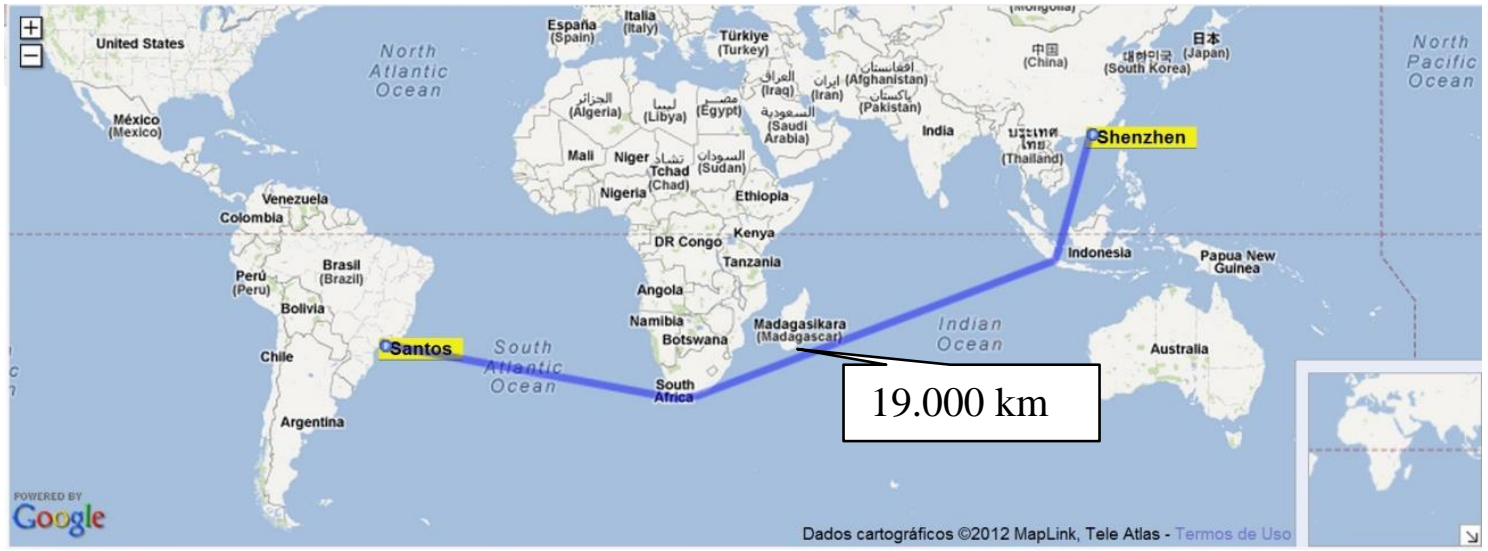

Fonte: Sea-Rates (2015).

\section{Emissões de transporte terrestre}

Para se calcular a emissão incorporada de carbono no transporte dos materiais pesquisados,é necessário se multiplicar a distância percorrida com carga por um fator de emissão (FE) de carbono.

No caso do transporte rodoviário por caminhão a diesel, foram identificadas duas fontes de informação diferentes:

(a) um artigo de referência do Proclima ${ }^{3}$

(ÁLVARES JUNIOR; LINKE, 2015); e

(b) um artigo da Esalq ${ }^{4}$ (BARTHOLOMEU;

CAIXETA FILHO, 2008).

${ }^{3}$ O Proclima é o Programa Estadual de Mudancas Climáticas

Globais do Estado de São Paulo. O programa é coordenado pela Divisão de Mudanças Climáticas da Companhia Ambiental do Estado de São Paulo (Cetesb).
O artigo do Proclima foi escrito pelo Gerente do Setor de Operação e Fiscalização da Cetesb (Fontes Móveis) e pelo Gerente da Divisão de Engenharia e Certificação da Cetesb (Fontes Móveis). Nesse artigo, foi utilizado o método Bottom-Up a partir de informações de referência de índices de emissões de motores a diesel europeus, considerados pelos autores como similares à realidade local. $\mathrm{O}$ artigo apresenta como fator de emissão o valor de $280 \mathrm{gCO}_{2} / \mathrm{km}$ (ÁLVARES JUNIOR; LINKE, 2015).

Para a presente análise foi necessário estabelecer um fator de conversão de $\mathrm{gCO}_{2} / \mathrm{t}$. km. Para tanto, assumiu-se uma capacidade de carga entre $10 \mathrm{t}$ e 14 t para um caminhão médio. Dessa forma, o

${ }^{4}$ Escola Superior de Agronomia Luiz de Queiroz, Universidade de São Paulo. 
fator de conversão resultante variaria entre 20 $\mathrm{gCO}_{2} / \mathrm{t} . \mathrm{km}$ e $28 \mathrm{gCO}_{2} / \mathrm{t} . \mathrm{km}$.

$\mathrm{O}$ artigo da Esalq apresenta os resultados de um trabalho realizado pelo Grupo de Pesquisa e Extensão em Logística Agroindustrial (EsalqLOG). Nesse artigo é realizado um estudo de caso para o cálculo do fator de emissão de carbono para caminhões a diesel no trajeto de Rondonópolis, RO, até Santos, SP. Nessa pesquisa foram encontrados valores de índices de emissões entre 26,5 $\mathrm{gCO}_{2} / \mathrm{t} . \mathrm{km}$ e 28,5 $\mathrm{gCO}_{2} / \mathrm{t} . \mathrm{km}$ para rotas consideradas melhores e piores pela referida pesquisa. A Tabela 2 compara os fatores de emissão de $\mathrm{CO}_{2}$ eq no transporte por caminhão a diesel das duas referências bibliográficas.

Comparando-se as duas fontes (ver Tabela 2) foi possível constatar a congruência entre ambas as abordagens. Para a presente pesquisa, foi escolhido o fator de conversão de $26,5 \mathrm{~g} \mathrm{CO}_{2} / \mathrm{t} . \mathrm{km}$, por dois motivos: esse valor foi calculado a partir de um estudo de caso real; e representa a média de emissão dos melhores trechos de estrada, ou seja, estradas com qualidade compatível com a que se espera dos trechos de transporte terrestre presumidos nesta pesquisa, devido ao fato de estarem, em grande parte, sob os cuidados de concessões rodoviárias privatizadas.

\section{Emissões de transporte marítimo}

Para o fator de emissão no transporte marítimo através de navio cargueiro de contêineres, foram identificadas quatro fontes de informação: (a) Wallenius Wilhelmsen Logistics

(WALLENIUS, 2015);

(b) Green Logistics (LEONARDI; BROWNIE, 2009);

(c) JF Hillebrand Global Beverage Logistics

(JFHILLEBRAND, 2015); e

(d) WMU Journal of Maritime (PSARAFTIS; KONTOVAS, 2015).

A Tabela 3 compara os fatores de emissão de $\mathrm{CO}_{2} \mathrm{eq}$ no transporte marítimo, a partir das referências bibliográficas.

Considerando-se as fontes pesquisadas, apenas duas (WMU Journal of Maritime e Green Logistics) não são empresas privadas. O artigo da WMU Journal of Maritime (PSARAFTIS KONTOVAS, 2015) cita um fator de emissão calculado por terceiros, ou seja, o relatório ambiental de uma empresa global de logística (MAERSKLINE, [2008]).

Dessa forma, foi escolhida como fonte para o fator de emissão a Green Logistics (LEONARDI; BROWNE, 2009), com valores provenientes de dois estudos de caso: $20 \mathrm{gCO}_{2} / \mathrm{t} . \mathrm{km}$ e 33 $\mathrm{gCO}_{2} / \mathrm{t} . \mathrm{km}$.

Para os cálculos deste estudo, foi definida como fator de emissão a média entre os valores de 20 $\mathrm{gCO}_{2} / \mathrm{t} . \mathrm{km}$ e $33 \mathrm{gCO}_{2} / \mathrm{t} . \mathrm{km}$, portanto 26,5 $\mathrm{gCO}_{2} / \mathrm{t} . \mathrm{km}$ (coincidentemente o mesmo valor do fator de emissão escolhido para o caminhão a diesel).

Tabela 2 - Fatores de emissão de $\mathrm{CO}_{2}$ eq para transporte realizado com caminhão a diesel

\begin{tabular}{lc}
\hline \multicolumn{1}{c}{ Fonte } & Fator de emissão $\left(\mathbf{g C O}_{2} / \mathbf{t . k m}\right)$ \\
\hline Proclima (ÁLVARES, 2012) & $20-28$ \\
\hline Esalq (BARTHOLOMEU, 2008) & $26,5-28,5$ \\
\hline
\end{tabular}

Tabela 3- Fatores de emissão de $\mathrm{CO}_{2}$ eq para o transporte marítimo em contêineres

\begin{tabular}{l|l|c}
\hline \multicolumn{1}{c|}{ Fonte } & \multicolumn{1}{c|}{ Comentário } & \multicolumn{1}{c}{\begin{tabular}{c}
\multicolumn{1}{c}{ Cator de emissão } \\
(gCO//t.km)
\end{tabular}} \\
\hline $\begin{array}{l}\text { WMU Journal of } \\
\text { Maritime (PSARAFTIS, } \\
\text { 2009) }\end{array}$ & $\begin{array}{l}\text { Artigo em publicação periódica da Universidade } \\
\text { Marítima da Suécia que cita o relatório ambiental de } \\
\text { uma empresa global de logística (MAERSKLINE, } \\
[2008]) .\end{array}$ & 11 \\
\hline $\begin{array}{l}\text { JF Hillebrand } \\
(\text { JFH, 2012) }\end{array}$ & $\begin{array}{l}\text { Calculadora de carbono de empresa global de logística } \\
\text { de bebidas. }\end{array}$ & \multirow{2}{*}{14} \\
\hline $\begin{array}{l}\text { Green Logistics } \\
\text { (LEONARDI, 2009) }\end{array}$ & $\begin{array}{l}\text { Artigo científico do grupo de pesquisa em logística } \\
\text { verde da Universidade de Cardiff, no Reino Unido }\end{array}$ & $20-33$ \\
\hline $\begin{array}{l}\text { Wallenius Wilhelmsen } \\
\text { Logistics } \\
(\text { WWL, 2010) }\end{array}$ & $\begin{array}{l}\text { Relatório ambiental e social de empresa global de } \\
\text { logística. }\end{array}$ & 31 \\
\hline
\end{tabular}

12 Hernandes, T. Z. 


\section{Cálculo do saldo de sequestro de carbono}

O cálculo do saldo de sequestro de carbono dos materiais é feito multiplicando-se a distância percorrida com cada carga por um fator de emissão de carbono expresso em gramas de $\mathrm{CO}_{2}$ eq emitidas por tonelada e por quilometro: $\mathrm{gCO}_{2} \mathrm{eq} / \mathrm{t} . \mathrm{km}$.

Dessa maneira, são obtidos os dados quantitativos apresentados na tabela comparativa (Tabela 4), em que são relacionadas as distâncias percorridas por uma carga hipotética de $1 \mathrm{t}$ de bambu e de $1 \mathrm{t}$ de eucalipto. Cada uma, segundo o trajeto estimado. Para cada distância e tipo de transporte, é feita a multiplicação pelo fator de emissão correspondente, de modo a estimar a quantidade de carbono emitida no transporte.

Depois de calculada a quantidade de $\mathrm{CO}_{2} \mathrm{eq}$ emitido no transporte de cada uma das cargas, é feita a subtração entre a quantidade de $\mathrm{CO}_{2} \mathrm{eq}$ incorporado em $1 \mathrm{t}$ de cada material. Essa diferença é o saldo de emissão de $\mathrm{CO}_{2}$ eq no transporte dos dois materiais. Assim, foi possível chegar aos dados apresentados na Tabela 5 e no gráfico da Figura 6.

Como resultado, é possível observar que, apesar de o transporte por longas distâncias não ser capaz sozinho de inviabilizar o potencial do sequestro de carbono do piso de bambu, suas emissões de transporte são significativas ao comprometer mais de um quarto do total de $\mathrm{CO}_{2}$ eq incorporado no material.

Para efeito de comparação, são calculadas as emissões para a aplicação de $1 \mathrm{~m}^{2}$ de cada uma das duas opções de acabamento de piso, bambu e piso maciço de eucalipto. A confrontação é possível, uma vez que as duas soluções de acabamento de piso são aplicadas sobre a mesma condição preexistente, ou seja, um contrapiso regularizado e nivelado.

Para o eucalipto, com o uso de réguas de $20 \mathrm{~mm}$ de espessura e densidade de $1.040 \quad \mathrm{~kg} / \mathrm{m}^{3}$ (INSTITUTO..., 2011), é alcançada uma massa de $20,4 \mathrm{~kg} / \mathrm{m}^{2}$. Para o piso de bambu, com réguas de $12 \mathrm{~mm}$ e $14 \mathrm{~mm}$ de espessura, as massas obtidas são de $8,56 \mathrm{~kg} / \mathrm{m}^{2}$ e $10 \mathrm{~kg} / \mathrm{m}^{2}$ respectivamente. A Tabela 6 ilustra o cálculo do saldo de emissão de $\mathrm{CO}_{2}$ eq para $1 \mathrm{t}$ de bambu e também para $1 \mathrm{t}$ de eucalipto.

Adicionalmente, esses dados são apresentados no gráfico da Figura 7, comparando-se $\quad \mathrm{O} \quad \mathrm{CO}_{2} \mathrm{eq}$ incorporado em $1 \mathrm{~m}^{2}$ de piso de bambu ante $1 \mathrm{~m}^{2}$ de piso de eucalipto e as respectivas emissões de $\mathrm{CO}_{2}$ eq no transporte de cada material.

Devido à característica de maior espessura do piso de eucalipto, seu potencial de sequestro de carbono é maior, em termos absolutos, por metro quadrado de piso acabado. Ainda, mesmo se utilizando maior massa por metro quadrado de piso acabado, a emissão de gases de efeito estufa relacionada ao transporte é cerca de 8 vezes menor quando comparada à do piso de bambu.

É importante enfatizar que não foram contabilizadas, dentro do escopo deste trabalho, as emissões referentes ao processamento dos materiais, devido à impossibilidade de identificar seus fornecedores.

Tabela 4- Tabela de emissão de $\mathrm{CO}_{2}$ pelo transporte de $1 \mathrm{t}$ de bambu ou eucalipto

\begin{tabular}{c|c|c|c|c|c}
\hline Material & Trajeto & Distância & Tipo & $\begin{array}{c}\text { Fator de emissão } \\
\mathbf{C O}_{2} / \mathbf{k m}\end{array}$ & $\begin{array}{c}\text { Total de C0 } \\
\text { para 1 t }\end{array}$ \\
\hline Bambu & Shenzhen-Santos & $\begin{array}{c}19.000 \\
\mathrm{~km}\end{array}$ & $\begin{array}{c}\text { Cargueiro } \\
\text { marítimo }\end{array}$ & $26,5 \mathrm{gCO}_{2} / \mathrm{t} . \mathrm{km}$ & $503,5 \mathrm{~kg}$ \\
\hline & Santos-S. J. do Rio Preto & $500 \mathrm{~km}$ & $\begin{array}{c}\text { Caminhão } \\
\text { a diesel }\end{array}$ & $26,5 \mathrm{gCO}_{2} / \mathrm{t} . \mathrm{km}$ & $13,2 \mathrm{~kg}$ \\
\hline \multicolumn{5}{|c|}{ TOTAL } & $516,7 \mathrm{~kg}$ \\
\hline Eucalipto & Plantio-São Paulo & $500 \mathrm{~km}$ & $\begin{array}{c}\text { Caminhão } \\
\text { a diesel }\end{array}$ & $26,5 \mathrm{gCO}_{2} / \mathrm{t} . \mathrm{km}$ & $13,2 \mathrm{~kg}$ \\
\hline & São Paulo-S. J. do Rio \\
Preto & $500 \mathrm{~km}$ & $\begin{array}{c}\text { Caminhão } \\
\text { a diesel }\end{array}$ & $26,5 \mathrm{gCO}_{2} / \mathrm{t} . \mathrm{km}$ & $13,2 \mathrm{~kg}$ \\
\hline
\end{tabular}

Tabela 5 - Cálculo do saldo de emissão de $\mathrm{CO}_{2}$ para $1 \mathrm{t}$ de bambu ou $1 \mathrm{t}$ de eucalipto

\begin{tabular}{lllll}
\hline & \multicolumn{1}{c}{ Bambu } & \multicolumn{1}{c}{$\%$} & \multicolumn{1}{c}{ Eucalipto } & \multicolumn{1}{c}{$\%$} \\
\hline Carbono incorp. em 1 t & $+1.835 \mathrm{~kg}$ & $100 \%$ & $+1.835 \mathrm{~kg}$ & $100 \%$ \\
Emissão no transporte & $-516,7 \mathrm{~kg}$ & $28,1 \%$ & $-26,4 \mathrm{~kg}$ & $1,4 \%$ \\
Saldo de sequestro & $+1.318,30 \mathrm{~kg}$ & $71,9 \%$ & $+1.808,6 \mathrm{~kg}$ & $98,6 \%$ \\
\hline
\end{tabular}


Figura 6 - Comparação entre o $\mathrm{CO}_{2}$ incorporado e o $\mathrm{CO}_{2}$ emitido no transporte de $1 \mathrm{t}$ de bambu ou de eucalipto

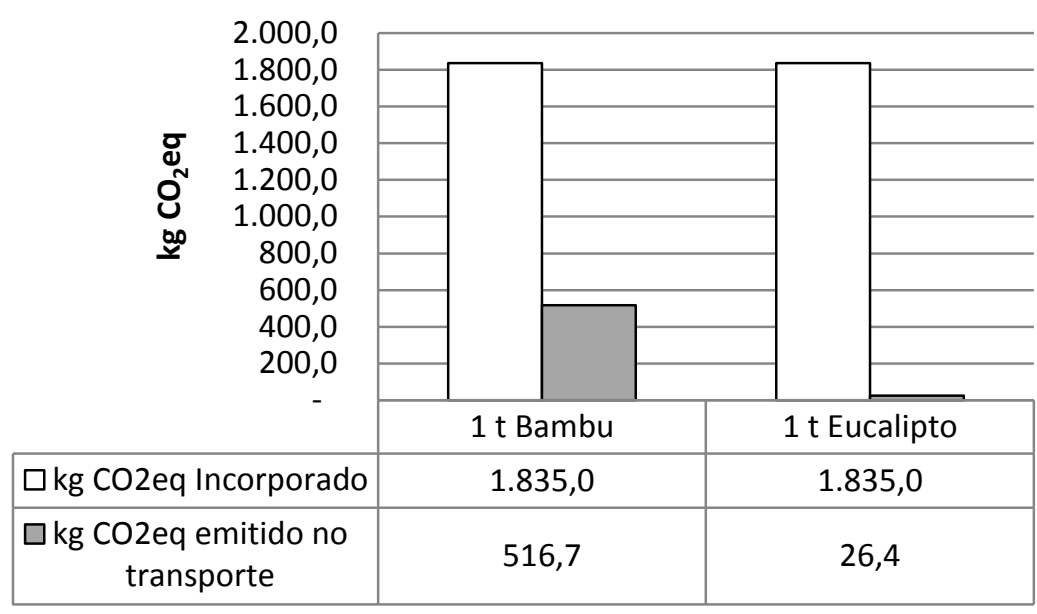

Tabela 6 - Cálculo do saldo de emissão de $\mathrm{CO}_{2}$ para $1 \mathrm{t}$ de bambu ou $1 \mathrm{t}$ de eucalipto

\begin{tabular}{lrrr}
\hline & Bambu & Bambu & Eucalipto \\
& $\mathbf{1 2} \mathbf{~ m m}$ & $\mathbf{1 4} \mathbf{~ m m}$ & \multicolumn{2}{c}{$\mathbf{~ c m}$} \\
\hline Peso de $1 \mathbf{~ m}^{\mathbf{2}}(\mathbf{k g})$ & 8,56 & 10,00 & 20,40 \\
$\mathbf{C O}_{2}$ incorporado $\left(\mathbf{k g C O}_{\mathbf{2}} \mathbf{e q}\right)$ & 15,72 & 18,35 & 37,43 \\
$\mathbf{C O}_{2}$ emitido no transporte $(\mathbf{k g C O} \mathbf{2} \mathbf{e q})$ & 4,42 & 5,20 & 0,52 \\
Saldo de sequestro $\left(\mathbf{k g C O}_{\mathbf{2}} \mathbf{e q}\right)$ & 11,30 & 13,15 & 36,91 \\
\hline
\end{tabular}

Figura 7- Comparação entre o $\mathrm{CO}_{2}$ incorporado e emitido no transporte para $1 \mathrm{~m}^{2}$ de piso

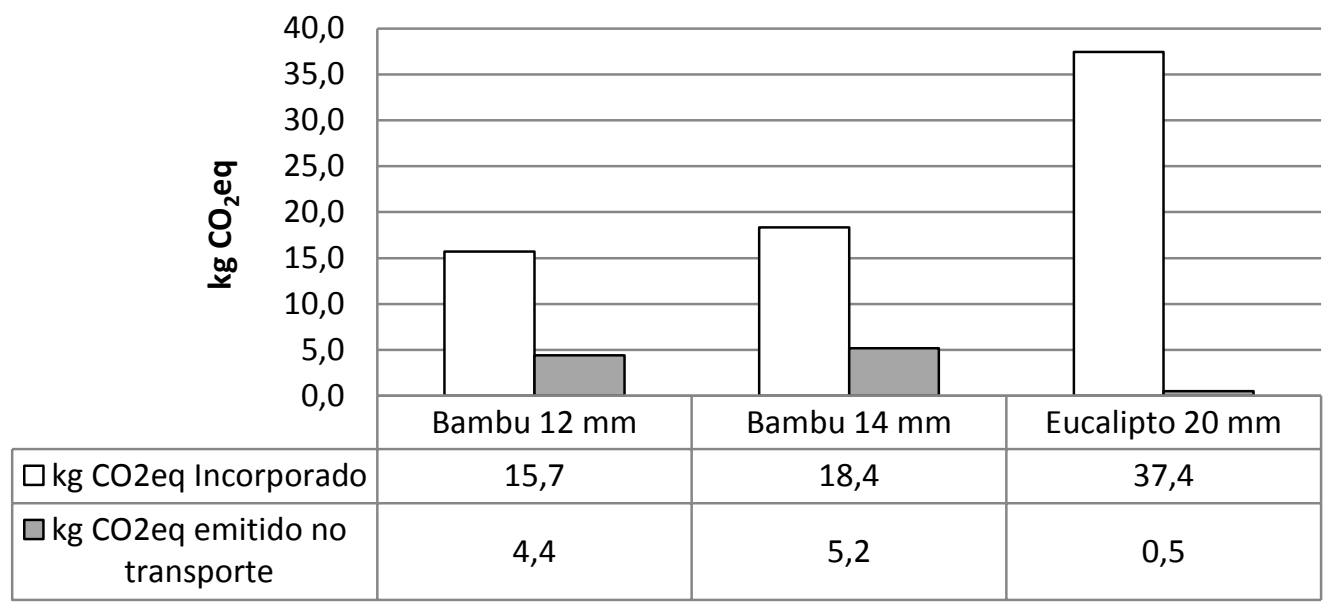

\section{Considerações finais}

A pesquisa demonstrou a dificuldade de se obterem informações sobre os fatores de emissão de $\mathrm{CO}_{2}$ para o transporte marítimo das cargas consideradas. A abordagem procurou ser consistente, analisando o maior número possível de fontes e procurando como referências trabalhos acadêmicos.
$\mathrm{O}$ cálculo da emissão de $\mathrm{CO}_{2}$ referente ao transporte demonstra que as distâncias percorridas podem comprometer de maneira significativa o potencial de sequestro de carbono de um material. Nesse caso, apenas o transporte foi capaz de comprometer $28,1 \%$ de todo o potencial de sequestro de carbono do piso de bambu chinês em comparação com $1,4 \%$ para o piso de eucalipto brasileiro. 
$\mathrm{O}$ saldo do sequestro de $\mathrm{CO}_{2}$ do piso de eucalipto não pode ser considerado individualmente como medida de seu potencial, uma vez que não foram contabilizadas as emissões em seu plantio e processamento. No entanto, a ordem de grandeza de seu saldo $\left(98,6 \%\right.$ de $\mathrm{CO}_{2}$ incorporado, depois de serem subtraídas as emissões do transporte) pode ser considerada como uma indicação promissora, levando-se em consideração que o processamento mecânico da madeira de eucalipto no Brasil faz uso principalmente de energia elétrica de uma matriz que apresenta uma significativamente baixa emissão de $\mathrm{CO}_{2}$.

Por fim, avaliando-se o piso de bambu importado da china, é possível afirmar que a etapa de transporte não consegue sozinha comprometer o total do saldo de carbono do material. No entanto, destaca-se que o cálculo do saldo de carbono do material - e portanto, a afirmação definitiva de sua vantagem ambiental - só será possível, obrigatoriamente, a partir de uma avaliação futura do ciclo de vida profunda e abrangente, incluindo também as etapas de produção do bambu e a fabricação do piso em seu país de origem.

Como perspectivas para a continuidade deste trabalho cita-se a possibilidade de confrontação dos dados obtidos com outros materiais de acabamentos para pisos ou, ainda, a consideração das etapas de produção e fabricação no âmbito da análise do ciclo de vida desses materiais.

\section{Referências}

ÁLVARES JUNIOR, O. de M.; LINKE, R. R. A. Metodologia Simplificada de Cálculo das Emissões de Gases do Efeito Estufa de Frotas de Veículos no Brasil. Programa Proclima da Secretaria Estadual do Meio Ambiente do Estado de São Paulo. Disponível em:

<http://www.ambiente.sp.gov.br/proclima/PDF/inv entario_efeitoestufa.pdf>. Acesso em: 14 jan. 2015.

\section{ASSOALHO ECOLÓGICO. [Página}

institucional]. Disponível em:

<http://www.assoalhoecologico.com.br/>. Acesso em: 14 jan. 2015.

ASSOCIAÇÃO BRASILEIRA DE PRODUTORES DE FLORESTAS PLANTADAS. Anuário Estatístico da ABRAF2011: ano-base 2010. Brasília: ABRAF, 2011.

BARTHOLOMEU, D. B.;CAIXETA FILHO, J. V.Impactos Econômicos e Ambientais Decorrentes do Estado de Conservação das Rodovias Brasileiras: um estudo de caso. Revista de Economia e Sociologia Rural, Piracicaba, v. 46, n. 3, p. 703-738, jul./set. 2008.
DELGADO, P. S. O bambu como material ecoeficiente: caracterização e estudos exploratórios de aplicações. 2011. 67 f. Dissertação (Mestrado em Engenharia de Materiais) - Universidade Federal de Ouro Preto, Ouro Preto, 2011.

ECORI. [Página institucional]. Disponível em: <http://www.ecori.com.br/>. Acesso em: 14 jan. 2015.

GOOGLE MAPS. [Imagem]. Disponível em: <http://maps.google.com.br>. Acesso em: 9 jun. 2012.

\section{INSTITUTO DE PESQUISAS}

TECNOLÓGICAS. Informações Sobre

Madeiras. 2011. Disponível em:

<http://www.ipt.br/consultas_online/informacoes_ sobre_madeira $>$. Acesso em: 24 set. 2012.

\section{JF HILLEBRAND. JF Hillebrand Global}

Beverage Logistics.Disponível em:

<http://www.jfhillebrand.com/DesktopModules/JF H.CarbonCalculator/Readme.htm>. Acesso em: 14 jan. 2015.

LEONARDI, J.; BROWNE, M. Method

Forassessing the Carbon Footprint of Maritime Freight Transport: european case study and results.In: ANNUAL LOGISTICS RESEARCH NETWORK CONFERENCE, 14., Cardiff, 2009. Proceedings... Cardiff, 2009.

\section{MAERSKLINE. Constant Care for de}

Environment. Denmark: Kopenhagen, [2008].

Disponível em:

<http://www.maerskline.com/globalfile/?path=/pdf /environmental_brochure>. Acesso em: 14 jan. 2015.

MARTINS, L. Entrevista Concedida ao Arq. Thiago Zaldini Hernandes no dia 16/04/2012, na loja da ECORI PISOS ECOLÓGICOS. São José do Rio Preto, 2012.

PSARAFTIS, H. N.; KONTOVAS, C. A. $\mathbf{C O}_{2}$ Emission Statistics for the World Commercial Fleet.World Maritime University (Sweden).WMU Journal of Maritime Affairs. Disponível em: <http://www.martrans.org/docs/publ/REFEREED $\%$ 20JOURNALS/WMUJMA\%20EMISSIONS $\% 2$ 02009.pdf>. Acesso em: 14 jan. 2015.

SEA-RATES. Port-to-Port Distances. Disponível em:

<http://www.searates.com/reference/portdistance/> . Acesso em: 14 jan. 2015. 
SILVA, D. A. L. Avaliação do Ciclo de Vida da

Produção do Painel de Madeira. Dissertação

(Mestrado em Engenharia Civil) - Escola de

Engenharia de São Carlos, Universidade

WALLENIUS WILHELMSEN LOGISTICS.

Environmental Sustainability Report 2010.

Disponível em:

<http://www.2wglobal.com/www/environment/esr

2010/Resources/WWL_ESR_2010.pdf>. Acesso

em: 14 ja. 2015.

\section{Revista Ambiente Construído}

Associação Nacional de Tecnologia do Ambiente Construído

Av. Osvaldo Aranha, $99-3^{\circ}$ andar, Centro

Porto Alegre - RS - Brasil

CEP $90035-190$

Telefone: +55 (51) 3308-4084

Fax: +55 (51) 3308-4054

www.seer.ufrgs.br/ambienteconstruido

E-mail: ambienteconstruido@ufrgs.br

16 Hernandes, T. Z. 